\title{
Neuroplasticidade na Educação e Reabilitação Cognitiva da Deficiência Intelectual
}

\author{
Neuroplasticity in Education and Cognitive Rehabilitation of Intellectual \\ Disability \\ Neuroplasticidad en la Educación y Rehabilitación Cognitiva de la Deficiencia \\ Intelectual
}

\author{
* Denise Oliveira Ribeiro \\ Mestranda pela Universidade Federal de Minas Gerais, Belo Horizonte, Minas Gerais, Brasil. \\ d3niseribeir0@gmail.com \\ ${ }^{* *}$ Patricia Martins de Freitas, \\ Professora doutora na Universidade Federal da Bahia, Vitória da Conquista, Bahia, Brasil. \\ pmfrei@gmail.com
}

Recebido em 02 de agosto de 2018

Aprovado em 15 de abril de 2019

Publicado em 05 de junho de 2019

\section{RESUMO}

$\mathrm{Na}$ deficiência intelectual os déficits no processamento cognitivo impõem limitações na funcionalidade desses indivíduos, impactando severamente os aspectos sociais, comportamentais e educacionais. A inclusão escolar cria a oportunidade de inserir as crianças com deficiências no ensino regular, mas as práticas pedagógicas utilizadas não têm permitido o desenvolvimento da alfabetização e de habilidades matemáticas, proporcionando apenas um ambiente adequado para socialização e recreação. De modo geral, a formação dos professores possui lacunas que estão relacionadas a pouco ou nenhum conhecimento das contribuições, ferramentas e estratégias das neurociências para adaptação curricular. O presente estudo tem como objetivo descrever brevemente o perfil funcional da deficiência intelectual, demonstrando técnicas que favorecem a adaptação curricular para essas crianças ao estabelecer a relação entre educação e neuroplasticidade. As intervenções psicoeducacionais precisam de um planejamento do currículo de forma individualizada, direcionando as atividades para as necessidades de cada aluno. A aplicação de técnicas comportamentais tem sido bastante eficiente no processo de aquisição de habilidades funcionais e escolares, disponibilizando uma série de ferramentas que favoreçam o desenvolvimento de atividades de vida diária e emergência de habilidades escolares como leitura, escrita e aritmética.

Palavras-chave: Deficiência intelectual; Educação inclusiva; Neuropsicologia. 


\section{ABSTRACT}

In intellectual disability deficits in cognitive processing impose limitations on the functionality of these individuals, severely impacting social, behavioral and educational aspects. School inclusion creates the opportunity to insert children with disabilities into regular education, but the pedagogical practices used have not allowed for the development of literacy and math skills, providing only a suitable environment for socialization and recreation. In general, teacher training has gaps that are related to little or no knowledge of neuroscience contributions, tools, and strategies for curricular adaptation. The present study aims to briefly describe the functional profile of intellectual disability, demonstrating techniques that favor curricular adaptation for these children when establishing the relationship between education and neuroplasticity. Educative interventions need curriculum planning in an individualized way, directing activities to the needs of each student. The application of behavioral techniques has been quite efficient in the process of acquisition of functional and scholastic abilities, providing a series of tools that favor the development of activities of daily life and emergence of school skills such as reading, writing, and arithmetic.

Keywords: Intellectual disability; Inclusive education; Neuropsychology.

\section{RESUMEN}

En la deficiencia intelectual los déficits en el procesamiento cognitivo imponen limitaciones en la funcionalidad de esos individuos, impactando severamente los aspectos sociales, comportamentales y educativos. La inclusión escolar crea la oportunidad de insertar a los niños con discapacidades en la enseñanza regular, pero las prácticas pedagógicas utilizadas no han permitido el desarrollo de la alfabetización y de las habilidades matemáticas, proporcionando sólo un ambiente adecuado para la socialización y la recreación. En general la formación de los profesores tiene lagunas que están relacionadas con poco o ningún conocimiento de las contribuciones, herramientas y estrategias de las neurociencias para adaptación curricular. El presente estudio tiene como objetivo describir brevemente el perfil funcional de la discapacidad intelectual, demostrando técnicas que favorecen la adaptación curricular para esos niños al establecer la relación entre educación y neuroplasticidad. Las intervenciones psicoeducativas necesitan una planificación del currículo de forma individualizada, dirigiendo las actividades a las necesidades de cada alumno. La aplicación de técnicas conductuales ha sido bastante eficiente en el proceso de adquisición de habilidades funcionales y escolares, ofreciendo una serie de herramientas que favorezcan el desarrollo de actividades de vida diaria y emergencia de habilidades escolares como lectura, escritura y aritmética.

Palabras clave: Deficiencia intelectual; Educación inclusiva; Neuropsicología. 


\section{Introdução}

As alterações do neurodesenvolvimento provocam déficits cognitivos, que geram impactos significativos na funcionalidade dos indivíduos. As crianças com atraso no desenvolvimento demonstram processos mais lentos na aquisição de habilidades e funções e por isso necessitam de programas de reabilitação. As limitações iniciais, como o atraso na aquisição da marcha ou da linguagem, podem dificultar a interação social e a inserção em diversos ambientes, principalmente no ambiente escolar (THAMBIRAJAH, 2011). Observa-se que o atraso significativo indica falha nos mecanismos de maturação cerebral. Muitos desses casos serão identificados com algum tipo de transtorno do desenvolvimento, sendo que quanto maiores os atrasos, maiores as chances de que a deficiência intelectual esteja presente (THAMBIRAJAH, 2011; OBRZUT; HYND, 2013).

A deficiência intelectual é o distúrbio do neurodesenvolvimento mais comum e prevalente (VASCONCELOS, 2004). Apesar da implantação do modelo educacional inclusivo, o processo educacional ainda é pouco efetivo. Os resultados alcançados são inexpressivos e mostram falhas na efetivação das políticas de inclusão (BARBOSA; MOREIRA, 2009). As crianças são matriculadas, mas permanecem na escola e não adquirem conhecimentos formais como a escrita, leitura e sofrem com a restrição da socialização com os pares (SILVA; CARVALHO, 2017; VELTRONE, 2008; PLETSCH, 2009). Conhecer as especificidades da deficiência intelectual e de outros transtornos do desenvolvimento, assim como, aplicar estratégias para adaptação do currículo escolar aumenta as chances de efetividade da inclusão escolar. Ao contrário disso, a inclusão tem sido marcada por diversas limitações, sendo o efeito da inserção da criança com deficiência intelectual no contexto da educação brasileira, muitas vezes, abaixo do esperado (SANTOS; MARTINS, 2015).

A perspectiva da inclusão escolar defende o direito de todos os alunos desenvolverem habilidades e competências necessárias no âmbito educacional como também fortalecer e ampliar suas potencialidades, permitindo a amplificação de conhecimentos prévios e a possibilidade de se adquirir novos saberes, mesmo quando há necessidade de adaptar as práticas educativas e a estrutura da escola às especificidades dos alunos com transtornos do neurodesenvolvimento (FREIRE, 2008; SANTOS, 2012; SOUZA; BATISTA, 2016). Na inclusão, as escolas vêm sendo constantemente desafiadas a pensar em currículos que contemplem o desenvolvimento escolar de crianças com desenvolvimento típico e também 
http://dx.doi.org/10.5902/1984686X31119

de crianças que possuem prejuízos nas mais diversas habilidades, como na linguagem, na motricidade, na percepção, na socialização, no comportamento, na orientação espacial e de assimilação de informações, sem que nenhum dos alunos esteja em desvantagem (SANTOS, MARTINS, 2015; PIMENTEL, 2013; SOUZA; GOMES, 2015).

As dificuldades e preconceitos são frequentes no processo de inclusão escolar de crianças com transtornos do neurodesenvolvimento, especialmente quando se trata da deficiência intelectual (SANTOS; MARTINS, 2015). Veltrone (2008), ao analisar a percepção de alunos com deficiência intelectual que estudavam em classes comuns de escolas regulares, percebeu que os estudantes veem o papel socializador da escola como uma vantagem da inclusão escolar, destacando como desvantagens, a dificuldade na apreensão do conteúdo e as situações de exclusão e estigmatização que sofrem por parte de seus colegas.

Tannous (2005), em um estudo realizado com professores da rede pública sobre a experiência de trabalhar com a inclusão de alunos com deficiência intelectual, verificou que os professores se percebem como despreparados e sem as ferramentas necessárias para realização adequada da inclusão escolar, possuem uma imagem preconceituosa sobre as capacidades de seus alunos, já que não acreditam na possibilidade de avanços significativos no processo de aprendizagem tal como desconhecem o significado da proposta de inclusão. No estudo de Barbosa e Duarte (2016) foram identificadas lacunas na formação profissional dos docentes como o pouco direcionamento para as especificidades da deficiência intelectual apesar dos professores participantes do estudo relatarem interesse na educação inclusiva.

Em sua pesquisa, Pletsch (2009) verificou o currículo, as práticas pedagógicas e o processo de ensino-aprendizagem das pessoas com deficiência intelectual. Os resultados indicam que os professores enfrentam obstáculos ao realizar o processo de alfabetização desses indivíduos mesmo, possuindo interesses e preocupações. O estudo apontou que, na maioria das vezes, os alunos apenas adquirem conhecimentos elementares que envolvem habilidades de cópia, recorte, pintura e colagem. A autora discute que as práticas curriculares para o ensino de alunos com deficiência intelectual são pouco eficientes, já que não promovem mudanças significativas na qualidade do ensino e nem disponibilizam um ambiente favorável para fortalecimento de potencialidades e emergência de oportunidades.

Ao considerarmos a complexidade da alfabetização e da aquisição das habilidades numéricas básicas, podemos verificar que para tais habilidades escolares são requeridas 
http://dx.doi.org/10.5902/1984686X31119

funções cognitivas que nas interações com o meio permitem a aplicação social. Em estudos de Ferreiro (2017), Teberosky e Palácio (1987) e Ferreiro (2001) verifica-se uma divisão entre a alfabetização como uma etapa de aquisição do sistema de decodificação grafema fonema e o uso nos diversos contextos sociais o letramento. Entretanto, os estudos em neurociências consideram que a alfabetização é o mecanismo que garante o processamento fonológico e lexical bem como o seu uso através da leitura fluente.

Nos estudos de Corso, Sperb e Salles (2013) e Refundini, Martins, Capellini e Dehaene (2011), foi demonstrado a relação entre alfabetização e componentes como a consciência fonológica e o processamento lexical. No presente estudo, a alfabetização assim como, os cálculos aritméticos são analisados como recursos de inserção social de indivíduos com deficiência intelectual, considerando a importância da aprendizagem garantindo não apenas a absorção de códigos arbitrários, mas o seu uso nos contextos sociais compreendendo o significado funcional e utilizando nas diversas situações do diaa-dia.

Considerando a importância da aprendizagem como um processo de aquisição de habilidades e competências que impliquem na alfabetização e letramento para o uso tanto dos códigos ortográficos quanto numéricos, portanto sejam significativamente eficazes e suficientes para atender as necessidades curriculares das crianças e jovens com a deficiência intelectual, diversos autores têm destacado a importância da abordagem multidisciplinar, considerando as contribuições das neurociências e educação (SILVA, 2010; PLETSCH; GLAT, 2012; PICKER; WALSH, 2013; SOUZA; GOMES, 2015; AINSWORTH et al., 2016; HAASE et al., 2016).

As habilidades escolares são focos de diversos estudos que buscam demonstrar suas especificidades para a aquisição de recursos que favorecem a inserção social. Os estudos em neurociências demonstram que a aquisição de habilidades escolares como leitura e aritmética ampliam de forma significativa as redes neurais, formando circuitos dinâmicos que contribuem com mecanismos de ativação mais amplos e associados com outras funções cognitivas como o planejamento, a tomada de decisão e controle inibitório (DEHAENE, 2011). As neurociências possuem uma natureza interdisciplinar que permitem o dialogo dos seus achados com a educação, especialmente para as crianças que possuem alguns transtornos do desenvolvimento como no caso da deficiência intelectual (CIASCA, et al. 2015; CONSEZA \& GUERRA, 2011) 
A interface com a neuropsicologia pode auxiliar no estabelecimento do perfil funcional de cada criança através de estratégias de avaliação cognitiva e do comportamento, direcionando o olhar para as potencialidades e dificuldades que cada aluno possui, o que permite ao professor avaliar quais estratégias pedagógicas seriam mais adequadas (FREITAS; CARDOSO, 2015). A neuropsicologia também utiliza de programas de treinamento cognitivo que estimula funções cognitivas deficientes, favorecendo o desempenho em habilidades requeridas no ambiente escolar, como leitura, escrita e aritmética. Os treinos também podem ser aplicados pelos professores, agilizando o processo de reorganização neurocognitiva (OBRZUT; HYND, 2013). Rossit e Goyos (2009), por exemplo, encontraram melhoras significativas na aprendizagem de matemática em crianças com deficiência intelectual através de um currículo baseado em elementos da Análise do Comportamento como o paradigma da equivalência de estímulos. Freitas et al . (2016) indicam que a utilização de avaliações funcionais e individualizadas, planejamentos curriculares direcionados para as especificidades e necessidades de cada criança e o ensino baseado em técnicas comportamentais são recursos que possibilitam uma adaptação curricular adequada aos alunos com esses déficits.

Esse artigo tem como objetivo oferecer um panorama sobre aspectos pedagógicos da educação especial, considerando o suporte de outras áreas do conhecimento como a neuropsicologia e psicologia comportamental. Assim, espera-se uma maior difusão de um modelo multidisciplinar para auxiliar a formação do conhecimento de profissionais que lidam com a educação de crianças com a deficiência intelectual associada. Neste ensaio será feita a relação entre deficiência intelectual, neuroplasticidade e educação especial.

\section{Desenvolvimento}

\section{Deficiência Intelectual e educação}

A deficiência intelectual é definida por evidentes déficits intelectuais verificados por testes de inteligência ou escalas de desenvolvimento que caracterizam as alterações no funcionamento psicossocial considerando o que é esperado para idade, escolaridade grupo social (MANNERKOSKI, et al., 2009). Essas limitações devem ser investigadas, utilizando os níveis apresentados pelo DSM-V: conceitual, prático e social (APA, 2014). funcionamento intelectual descreve a nossa capacidade para aprender e é associado a diferentes funções cognitivas, como raciocínio lógico, planejamento, resolução de problemas e pensamento abstrato, funções específicas e seus componentes interferem no 
http://dx.doi.org/10.5902/1984686X31119

desempenho global da cognição como, por exemplo, déficits de atenção, da memória de trabalho, do processamento lexical ou visuo-espacial, por isso devem ser amplamente investigadas.

Os comportamentos adaptativos resultam das dificuldades para apresentar comportamentos socioculturais estabelecidos em relação à independência em múltiplos ambientes (APA, 2014). Os níveis de severidade da deficiência intelectual são classificados em: leve, moderado, grave e profundo. Outra forma comumente usada para classificar o perfil cognitivo é a utilização de medidas do quociente de inteligência, sendo: leve (QI de 50 a 70); moderado (QI de 35 a 50); grave (QI de 20 a 35) e profundo (QI abaixo de 20) (APA, 2014).

Quanto mais leve o grau da deficiência intelectual, maior o potencial para uma vida independente, como escolarização em instituições de ensino regulares e socialização com os pares. Indivíduos com classificação moderada possuem capacidades para inserção no mercado de trabalho desde que auxiliado e supervisionado e tendo passado por processos de reabilitação e aprendizagem. Para o perfil grave, embora apresentem déficits comunicativos, podem compreender relativamente à linguagem e realizar procedimentos de autocuidado. Entretanto, aqueles que se encontraram na classificação profunda, são dependentes para atividades de vida diárias e dificilmente desenvolvem a comunicação (MENDONÇA, 2002).

A epidemiologia da deficiência intelectual aponta que o quadro atinge 1 a $2 \%$ da população mundial e é mais frequente em meninos (GALABURDA, 2010; MILIAN et al., 2013). A deficiência intelectual apresenta maiores taxas em países em desenvolvimento e em populações de baixa renda, indicando implicações para a saúde pública, que deve elaborar estratégias para favorecer a diminuição dessa prevalência (LLOYD; KENNEDY, 2014). Considerando que as variáveis preditoras da alta prevalência da deficiência intelectual são o manejo do parto, cuidado pré e pós-natal, insegurança alimentar, é necessário aumentar a melhoria no cuidado da saúde materno-infantil (MAULIK et al., 2011). Deve-se considerar que países em desenvolvimento apresentam menos ofertas em serviços de estimulação precoce (MAULIK et al., 2011). Tais serviços são importantes, pois podem reduzir a gravidade da deficiência intelectual, estimulando desde os primeiros meses de vida. Os estudos são convergentes com os efeitos positivos da reabilitação precoce que aumentam as chances de aquisição de funções e aperfeiçoamento cognitivo 
em crianças com deficiência intelectual (THAMBIRAJAH, 2011; OBRZUT; HYND, 2013; GULYAEVA, 2017).

As causas etiológicas da deficiência intelectual são heterogêneas. O quadro pode ser causado por fatores genéticos e/ou ambientais (GILISSEN et al., 2014). Por exemplo, disfunções neurocognitivas resultam de alterações sinápticas que depende de processos bioquímicos para transmissão da informação. Essa falha tem como base a expressão de proteínas que podem estar alteradas por falhas no código genético (GILISSEN et al., 2014).

Essa caracterização clínica está relacionada às alterações cognitivas, que são ocasionadas pelos danos neuronais múltiplos e, portanto, interferem na organização de diferentes funções. As funções que, em geral, apresentam desempenho abaixo do esperado na deficiência intelectual são: memória de trabalho, capacidade de planejamento e de resolução de problemas, controle atencional e velocidade de processamento. A falha no funcionamento de tais funções dificulta a aprendizagem de coisas novas, sendo necessário o uso de estratégias educativas especiais (FREITAS et al., 2016).

Então, o primeiro passo deve ser traçar o perfil neuropsicológico apresentado pela criança, com objetivo de identificar os déficits a partir de um modelo que compreenda os aspectos operacionais do sistema cognitivo, organizados em funções específicas, sendo que tais funções também possuem componentes específicos (OBRZUT; HYND, 2013). Quanto mais detalhes possuirmos sobre o tipo de déficit que as crianças apresentam, maior será precisão sobre o tipo de intervenção oferecida a ela. O segundo passo será organizar o currículo, que nada mais é que um programa de reabilitação ou estimulação cognitiva aplicado no contexto escolar (SNELL et al., 2010).

A avaliação neuropsicológica contribui para o mapeamento funcional, fundamentado na identificação de déficits específicos. Dentre os diversos aspectos avaliados, a inteligência faz parte do ponto de partida da avaliação, pois, a partir desse ponto é possível identificar a gravidade da deficiência, ou seja, o nível de comprometimento. Essa informação inicial auxilia na estimativa de um prognóstico, que não é exato, mas que permite a definição das perspectivas futuras da criança, orientando adequadamente a família. A avaliação também contribui para se ter medidas comparativas de antes e depois da intervenção, o que facilita a análise da evolução e dos ganhos obtidos pela criança, a partir das intervenções. Portanto, ressaltamos a importância da avaliação neuropsicológica, que pode ser realizada por profissionais de diversas áreas de atuação, incluindo educadores (OBRZUT; HYND, 2013). 


\section{Neuroplasticidade, Reabilitação e Educação}

A relação entre educação e estimulação cognitiva tem sido pouco estabelecida. A educação formal está estruturada em currículos que desenvolvem habilidades e competências formais para lidar com conhecimentos que não são adquiridos espontaneamente, porém os professores desconhecem os processos neurocognitivos que são recrutados para a aprendizagem. O processo de aprender envolve um conjunto de funções cognitivas altamente complexas, que estão em desenvolvimento, o que caracteriza educação formal como um mecanismo bidirecional no qual a estimulação gera melhor desempenho neurocognitivo que demanda mais estímulos.

O cérebro em desenvolvimento recebe estimulação complexa e sistemática no ambiente escolar, que aprimora as funções cognitivas dentro de um mecanismo de retroalimentação. As crianças que recebem estimulação cognitiva escolar adequada ao seu nível de desenvolvimento ampliarão a capacidade de resolver problemas de forma mais eficiente e adaptativa. A partir desta relação entre educação estimulação cognitiva é possível fazer um paralelo entre educação e reabilitação cognitiva. As crianças com deficiência intelectual têm no processo de escolarização um poderoso mecanismo para potencializar o seu desenvolvimento neurocognitivo. Na reabilitação cognitiva o resultado esperado é desenvolvimento de funções que estão deficientes por isso é necessário que a criança seja estimulada e ensinada intencionalmente.

Para compreendermos os efeitos da estimulação cognitiva como um é necessário entendermos o mecanismo neuronal da aprendizagem. A neuroplasticidade é o processo subjacente à estimulação cognitiva e às atividades realizadas pelo educador. A neuroplasticidade é definida como a capacidade do sistema nervoso de alterar sua estrutura ou função de acordo com as influências ambientais em que o indivíduo é exposto demonstrando $\mathrm{o}$ alto poder adaptativo da estrutura neuronal (AYDIN et al., 2007; BERMUDEZ et al., 2008; MARTINEZ-MORGA; MARTINEZ, 2016). O processo da aprendizagem corresponde há uma série de mecanismos neurofisiológicos que engloba a formação de novas conexões sinápticas, o crescimento de espinhas dendríticas, mudanças de conformação de macroproteínas nas membranas pós-sinápticas e também o aumento ou diminuição de neurotransmissores em áreas sinápticas funcionais (GULYAEVA, 2017). Aprendizagem é, portanto, a construção, manutenção e renovação de uma rede neural. Os 
http://dx.doi.org/10.5902/1984686X31119

mecanismos de mudança da estrutura são reflexos da interação entre a estimulação ambiental e o amadurecimento neuronal (JOHNSTON, 2009).

A neuroplasticidade vem sendo demonstrada em por diferentes eixos de pesquisa, como a recuperação após lesão cerebral e cirurgia de hemisferectomia (DE BODE et al., 2005; SADATO et al., 2004). Nessas condições a estrutura cerebral apesar de perdas significativas de tecido, mantém a capacidade para desenvolver as habilidades motoras e da linguagem. Outro conjunto de evidências vem a partir de estudos de neuroimagem funcional, utilizando a morfometria por voxel que permite verificar medidas de volume e espessura de substância cinzenta e branca em áreas específicas (BERMUDEZ et al., 2008). Através dessa tecnologia robusta, têm sido verificadas diferenças estruturais após exposição de aprendizagem, por exemplo, verificou-se diferenças com aumento da substância cinzenta nas áreas motoras, auditivas e visuo-espaciais, comparando músicos profissionais, iniciantes e não músicos, também foi verificada diferenças entre a estrutura de analfabetos e alfabetizados. Nesse eixo de evidências é possível verificar um efeito da exposição ambiental em mecanismos de aprendizagem sistemática, enfatizando como a prática sistemática causa neuroplasticidade estrutural. Além disso, a plasticidade neuronal vem sendo investigada considerando o estilo de vida e os efeitos para reduzir as perdas que são provocadas pelo avanço da idade. A importância desses estudos para a educação de pessoas com deficiência intelectual é justamente porque eles apontam que um dos mecanismos de manutenção da plasticidade é o engajamento cognitivo associado a atividade física e dieta saudável. A combinação dessas variáveis aumenta o potencial de manutenção das células neurais e proliferação sináptica em algumas regiões como áreas hipocampais melhorando o desempenho cognitivo, especialmente para a memória (POWER; SCHLAGGAR, 2017; PHILLIPS, 2017; VOSS et al., 2013).

O conjunto de evidências atuais confirma a lei de Hebb sobre quanto mais ativa uma rede sináptica mais forte, ela se torna e, portanto, mais facilmente será novamente ativa que significa plasticidade dependente de atividade (GANGULY; POO, 2013). Isso é o que o ocorre neurocognitivamente quando aprendemos algo. A neuroplasticidade molda a estrutura cerebral através de variáveis ambientais. As mudanças estruturais no cérebro podem ocorrer por meio da aprendizagem, levando a reorganização de redes neuronais. Essas modificações são mais percebidas em casos de alterações na estrutura ocasionadas por condições clínicas, sejam elas de causas orgânicas ou adquiridas, subjacentes aos transtornos do neurodesenvolvimento (ALTERMARK, 2014). A presença de uma alteração 
http://dx.doi.org/10.5902/1984686X31119

no desenvolvimento cerebral poderá levar a reorganização estrutural, garantindo a preservação e o desenvolvimento das funções cognitivas (JOHNSTON, 2009).

Os investimentos em reabilitação cognitiva são sustentados pelo argumento da natureza plástica do cérebro e pelo potencial de aprendizagem. Destaca-se a importância do início precoce das intervenções e programas de estimulação cognitiva a fim de aproveitar o período em que a estrutura está mais suscetível a essas modificações, a infância. Em relação à deficiência intelectual, a melhor estratégia de reabilitação é o uso de programas educacionais elaborados a partir de modelos de aprendizagem diretiva e individualizada. Associar a reabilitação cognitiva à educação formal é a forma eficiente de potencializar a estimulação cognitiva, que em geral é realizada durante uma ou duas horas semanais. Dentro de um programa educacional aplicado no ambiente escolar o tempo de estimulação aumenta para pelo menos 15 horas. O conhecimento da capacidade plástica do cérebro é indispensável para profissionais que trabalham com o processo de desenvolvimento de funções cognitivas em crianças, essencialmente para crianças que demandam maiores investimentos pedagógicos, como é o caso da deficiência intelectual. Um dos impactos ocasionados pela compreensão dos mecanismos de neuroplasticidade é a percepção do potencial de aprendizagem apesar da deficiência intelectual. Assim, no contexto da educação especial, o educador torna-se um profissional da reabilitação cognitiva atuando na reorganização neurocognitiva e favorecendo o desenvolvimento (MARTINEZ-MORGA; MARTINEZ, 2017).

O processo de educação das pessoas com deficiência intelectual deve considerar os princípios da neuroplasticidade. Assim, como na reabilitação, a neuroplasticidade é o pilar de sustentação, para que possamos aplicar intervenções, considerando que esses procedimentos podem melhorar a funcionalidade apresentada pelas crianças.

\section{Ensino individualizado - Adaptações}

A aquisição de habilidades escolares básicas, como leitura, escrita e aritmética, é essencial para que as crianças com deficiência intelectual possuam um futuro vocacional e alcancem a maior independência possível (SNELL et al., 2010). Para isso, deve ser feitas adaptações individualizadas considerando as necessidades e capacidades de cada criança. Adaptar o currículo para aprendizagem de uma criança com deficiência intelectual é estabelecer situações de aprendizagem capazes de estimular e de promover o desenvolvimento cognitivo, emocional e social. 
http://dx.doi.org/10.5902/1984686X31119

O que de fato muda com a detecção da deficiência intelectual e seus respectivos déficits é a necessidade de atendimento diferenciado, utilizando estratégias educacionais individualizadas, currículos adaptados para as necessidades de cada um e o uso de técnicas de ensino e aprendizagem com evidências científicas. O conhecimento sobre o desenvolvimento neurocognitivo e as inúmeras evidências sobre o potencial plástico da estrutura cerebral impulsionam os investimentos em programas de educação, reconhecendo-os como principal forma de reabilitação para a deficiência intelectual (GUSTAVSSON; KITTELSAA; TOSSEBRO, 2017).

A aprendizagem de crianças com deficiência intelectual ocorre de forma lenta, com baixa velocidade no regaste de informações armazenadas, permitindo a fixação de um número limitado de estímulos. Essas limitações podem ser resultado de estratégias educativas que não considerem o processo de individualização, ou seja, não consideram as especificidades cognitivas e comportamentais de cada criança (CARR et al., 2016).

Para que o processo de aquisição da aprendizagem obtenha resultados significativos, é necessário utilizar como base um modelo diretivo circunscrito aos alvos de aprendizagem como o intuito de facilitar a fixação e generalização do conteúdo aprendido (LLOYD; KENNEDY, 2014). O tempo que uma criança com deficiência intelectual leva para aprender é maior e quanto mais inespecífico o assunto trabalhado maior a será a lentidão na aquisição do conteúdo. Uma solução para essa questão seria utilizar atividades que facilitem a resposta passo-a-passo e que não tem foco em alvos amplos (CARR, et al. 2016).

Para a estruturação do currículo, devemos considerar o desenvolvimento de habilidades escolares básicas para crianças com deficiência intelectual leve e moderada. O currículo deve ser individual, de acordo com o que cada criança apresenta. Os déficits cognitivos são detectados pela avaliação neuropsicológica, procedimento que envolve aplicação de diferentes procedimentos como testes, tarefas cognitivas, escalas, observações, entrevistas com a família realizando uma ampla coleta de dados para verificar a função ou componente cognitivo que deverá estimulado. As tarefas que envolvem o componente cognitivo a ser treinado são exercitadas repetitivamente.

Na definição das metas curriculares, os objetivos são hierarquicamente organizados. Antes de aprender a comer sozinho, é necessário treinar o sentar, posteriormente o pegar no garfo, e, por fim, levar à boca. Cada uma dessas respostas é definida como metas de aprendizagem e trabalhada de forma sistemática. Outro elemento importante é orientar a 
http://dx.doi.org/10.5902/1984686X31119

família a trabalhar em casa as habilidades que estão sendo foco de aprendizagem na escola. Para isso é necessário que a família aprenda as técnicas de aprendizagem. A família deverá trabalhar com o comportamento fracionado e não com o comportamento alvo. Muitas famílias têm dificuldades de ensinar comportamentos, pois utilizam a instrução verbal para realizar o comportamento alvo. $\mathrm{O}$ uso de instruções verbais sobrecarrega a memória de trabalho, gerando a perda de informações e falha na execução do comportamento esperado.

A criança com deficiência intelectual deve receber tarefas para casa, sob a forma de situações aplicadas no dia-a-dia e não folhas de papel que, muito provavelmente, não fazem sentido para a sua rotina (AINSWORTH et al., 2016). As tarefas de lápis e papel não são reconhecidas pela família como algo que criança possa executar, aumentando o desinvestimento da família no processo educacional e interferindo no desenvolvimento escolar. Para treinar a escrita, pode-se pedir à criança para escrever um bilhete; para treinar a leitura, pode-se pedir à criança que leia o folheto de propaganda do supermercado; para treinar o comer sozinho, pode ser oferecida a refeição preferida da criança; para treinar o uso do banheiro, pode-se colocar música, criando um contexto para a criança estar lá.

Os métodos de aprendizagem do comportamento são utilizados também para a aprendizagem de habilidades escolares. Assim, para que a criança aprenda a nomear objetos, podem ser apresentadas duas figuras, uma delas terá o nome falado pelo professor; quando a criança olha ou pega essa figura, o professor deverá reforçar essa resposta. Esse procedimento deve ser feito de forma sistemática. O próximo passo é a criança segurar a figura, mostrar ou nomear, dependendo do seu nível de desenvolvimento cognitivo. Esse é um exemplo de como trabalhar o desenvolvimento funcional da linguagem/comunicação no contexto escolar.

O foco na instrução orienta que os comandos sejam dados de forma curta, utilizando apenas um verbo para favorecer a compreensão e não sobrecarregar a memória. Apenas um comando de cada vez. O domínio da aprendizagem se refere à sistemática da repetição, para garantir a aquisição e a automatização. A correção de erros é feita enfatizando o comportamento ou a resposta correta. A prática consiste em envolver os diversos ambientes da criança, aumentando o potencial para a generalização da resposta e, portanto, a funcionalidade. Os treinos de discriminação reforçam o que foi aprendido, através da complexidade do raciocínio de discriminar entre um estilo e outro. Por fim, a revisão cumulativa significa que, antes de iniciar uma nova meta de aprendizagem, o 
http://dx.doi.org/10.5902/1984686X31119

educador deve revisar o que foi aprendido (KNIGHT et al., 2013; VELTRONE; MENDES, 2011).

A aplicação de técnicas comportamentais tem sido bastante eficiente no processo de aquisição de habilidades funcionais e escolares, em crianças com deficiência intelectual (HARRIS; GRAHAM, 2003; ELIAS et al., 2008; AINSWORTH, 2016; COSTA; PICHARILLO; ELIAS, 2017). A partir da análise funcional do comportamento, o educador verifica que uma mesma resposta como, por exemplo, o "choro" pode ter diferentes contingências e, portanto, pode ser diferente. Para definir um comportamento, é importante analisar o contexto em que a resposta acontece e quais são as consequências que ocorrem depois da resposta. Em outras palavras, é preciso identificar a funcionalidade da resposta de choro da criança. Essa ferramenta também será utilizada para o planejamento de programas de aprendizagem. Para as situações em que se deseja aumentar a frequência do comportamento, deve-se aplicar a modelagem. Essa técnica é muito eficiente, se aplicada de forma adequada. A partir dela, o comportamento alvo deve ser fracionado. Por exemplo, para a criança executar uma tarefa de cópia de um desenho, primeiro devemos verificar se ela possui habilidades como manter-se sentada, pegar o lápis e posicioná-lo no papel. $O$ primeiro passo deverá ser treinar o sentar; posteriormente, pegar e segurar o lápis e, posteriormente, riscar o papel, para só depois fazer a cópia, utilizando um modelo (HARRIS; GRAHAM, 2003; OBRZUT; HYND, 2013).

O treino de equivalência de estímulos tem sido utilizado no ensino de algumas disciplinas escolares, mostrando resultados significativos para a aprendizagem de leitura, escrita e matemática (ROSSIT; GOYOS, 2009; SANTOS, 2012; OLIVEIRA; PENARIOL; GOYOS, 2013). Nesse modelo, a aprendizagem se dá através da rede de relações, representando um conjunto de estímulos e desempenhos. As relações de equivalência emergem, do ensino de discriminações condicionais, sendo o matching-to-sample o procedimento utilizado para instalação de discriminações condicionais (STROMER; MACKAY; STODDARD, 1992; ROSSIT; ZULIANI, 2003). Sidman (1971) descreveu essa relação da seguinte forma: apresenta-se um estímulo arbitrário ou modelos, outros estímulos devem ser apresentados sendo que apenas um demonstra equivalência com o modelo. Se o estímulo escolhido corresponder ao correto, a resposta deverá ser reforçada (SANTOS, 2012).

Um exemplo de como a equivalência de estímulos se aplica foi demonstrada por Llausas (2008) que estabeleceu a relação entre palavras e figuras ditadas e entre palavras 
ditadas e palavras impressas. Após treino, verificou a emergência da leitura compreensiva, escrita compreensiva e leitura expressiva. Rossit e Goyos (2009) por sua vez, utilizando a equivalência de estímulos para estimulação de habilidades matemáticas em indivíduos com deficiência intelectual e obtiveram resultados positivos. Os resultados dessas pesquisas evidenciam que o ensino de discriminações condicionais é um método favorável ao ensino de leitura, escrita e matemática, mostrando-se como um recurso para a prática pedagógica.

\section{Conclusão}

O presente artigo apresenta uma relação entre educação e reabilitação cognitiva demonstrando importância do conhecimento sobre o desenvolvimento neurocognitivo para os professores que atuam com a educação de crianças com a deficiência intelectual. A aplicação de um modelo inclusivo efetivo ainda carece de uma formação pedagógica mais dinâmica, multidisciplinar e rigorosamente científica. Esse problema já foi detectado no estudo de Barbosa e Moreira (2009) no qual os autores demonstraram a necessidade de mais cientifidade na estruturação de uma educação especial que seja inclusiva.

A compreensão dos aspectos neurocientíficos inclui entender que as funções cognitivas resultam da interação com o meio, os mecanismos sociais são moduladores das estruturas cerebrais. Portanto, é necessário que os educadores conheçam a dinâmica do funcionamento e das possibilidades de recuperação para estruturas que por diversas razões apresentam alterações do neurodesenvolvimento. A criança com deficiência intelectual não representa uma condição de completa incapacidade e precisa de ter a oportunidade do desenvolvimento escolar. Como contribuição o presente estudo destaca que o currículo escolar e a reabilitação da deficiência intelectual são procedimentos sedimentados nas evidências do potencial de neuroplasticidade, principalmente na infância. A aplicação de técnicas mais diretivas e individualizadas favorecem os ganhos funcionais, aumentando a qualidade de vida da criança. É importante consideramos os direitos educacionais das crianças com deficiência intelectual e os efeitos positivos que a escolarização exerce, sendo o principal mecanismo de aquisição de funções que favorecem a inserção social, aumento do nível de independência e a inserção profissional.

Assim, as características neurocognitivas da deficiência intelectual contribuem para a elaboração dos currículos escolares individualizados e orientados para estimular as funções cognitivas que favoreçam o desenvolvimento, sendo as habilidades escolares básicas, consequência da estimulação cognitiva. A partir desse ensaio consideramos a importância 
http://dx.doi.org/10.5902/1984686X31119

da maior difusão das especificidades neurocognitivas da deficiência intelectual e da formação em estratégias de ensino baseadas nas técnicas comportamentais como uma proposta de formação de professores que atuam na educação especial e inclusiva.

\section{Referências}

AINSWORTH, M. et al. Teaching phonics to groups of middle school students with autism, intellectual disabilities and complex communication needs. Research in Developmental Disabilities, v. 56, p. 165-176, 2016.

ALTERMARK, N. The ideology of neuroscience and intellectual disability: reconstituting the 'disordered' brain. Disability \& Society, v. 29, n. 9, p. 1460-1472, 2014.

AMERICAN PSYCHIATRIC ASSOCIATION. Manual Diagnóstico e Estatístico de Transtornos Mentais DSM-V. Porto Alegre: Artmed, 2014.

AYDIN, K. et al. Increased gray matter density in the parietal cortex of mathematicians: a voxel-based morphometry study. American Journal of Neuroradiology, v. 28, n. 10 p. 1859-1864, 2007.

BARBOSA, A. J. G.; MOREIRA, P. S. Deficiência mental e inclusão escolar: produção científica em Educação e Psicologia. Rev. bras. educ. espec., v. 15, n. 2, p. 337-352, 2009.

BARBOSA, M. O.; DUARTE, L. N. Deficiência intelectual e escolarização: uma análise das produções científicas. Comunicações, v. 23, n. 3, p. 351-368, 2016.

BERMUDEZ, P. et al. Neuroanatomical correlates of musicianship as revealed by cortical thickness and voxel-based morphometry. Cereb Cortex, v. 19, n. 7, p. 1583-1596, 2009.

CARR, Alan et al. The Handbook of Intellectual Disability and Clinical Psychology Practice. 2. ed. Londres: Routledge, 2016.

CIASCA, S. et al. Transtornos de aprendizagem: neurociência e interdisciplinaridade. Ribeirão Preto: Book Toy, 2015.

COSENZA, R. M.; GUERRA, L. B. Neurociência e educação: como o cérebro aprende. Porto Alegre: Artmed, 2011.

CORSO, H. V.; SPERB, T. M.; SALLES, J. F. Leitura de palavras e de texto em crianças: efeitos de série e tipo de escola, e dissociações de desempenhos. Letras de Hoje, v. 48, n. 1, p. 81-90, 2013.

COSTA, A. B.; PICHARILLO, A. D. M.; ELIAS, N. C. Avaliação de habilidades matemáticas em crianças com síndrome de Down e com desenvolvimento típico. Ciência \& Educação, Bauru, v. 23, n. 1, p. 255-272, 2017.

DE BODE, S. et al. Residual motor control and cortical representations of function following hemispherectomy: effects of etiology. Journal of Child Neurology, v. 20, p. 64-75, 2005.

DEHAENE, S. The massive impact of literacy on the brain and its consequences for education. Human Neuroplasticity Education, v. 117, p. 19-32, 2011. 
ELIAS, N. C. et al. Teaching manual signs to adults with mental retardation using matchingto-sample procedures and stimulus equivalence. The Analysis of verbal behavior, v. 24, n. 1, p. 1-13, 2008.

FERREIRO, E. Alfabetização em processo. São Paulo: Editora Cortez, 2017.

FERREIRO, E. Cultura escrita e educação: conversas de Emilia Ferreiro com José Antonio Castorina, Daniel Goldin e Rosa MariaTorres. Porto Alegre: ARTMED, 2001.

FREIRE, S. Um olhar sobre a inclusão. Revista da Educação, v. 15, n. 1, p. 5-20, 2008.

FREITAS, P. M. et al. Adaptações curriculares para crianças com deficiência intelectual moderada: contribuições da neuropsicologia do desenvolvimento. Pedagogia em Ação, Belo Horizonte, v. 8, n. 2, 2016.

FREITAS, P. M.; CARDOSO, T. G. S. Contribuições da Neuropsicologia para a inclusão educacional: como enfatizar as potencialidades diante das deficiências? Aprender Cadernos de Filosofia e Psicologia Educacional, Vitória da Conquista, v. 9, n. 15, p. 15173, 2015.

GALABURDA, A. Neuroscience, Education, and Learning Disabilities. Human Neuroplasticity and Education, Vaticano, v. 27, n. 151, p. 151-166, 2010.

GANGULY, K.; POO, M. Activity-dependent neural plasticity from bench to bedside. Neuron, v. 80, n. 3, p. 729-741, 2013.

GILISSEN, C. et al. Genome sequencing identifies major causes of severe intellectual disability. Nature, v. 511, n. 7509, p. 344-347, 2014.

GULYAEVA, N. V. Molecular mechanisms of neuroplasticity: An expanding universe. Biochemistry, Moscou, v. 82, n. 3, p. 237-242, 2017.

GUSTAVSSON, A.; KITTELSAA, A.; TØSSEBRO, J. Successful schooling for pupils with intellectual disabilities: the demand for a new paradigm. European Journal of Special Needs Education, v. 32, n. 4, p. 469-483, 2017.

HAASE, V. G. et al. Como a neuropsicologia pode contribuir para a educação de pessoas com deficiência intelectual e/ou autismo? Pedagogia em Ação, Belo Horizonte, v. 8, n. 2, 2016.

HARRIS, K. R.; GRAHAM, S. Self-regulated strategy development in the classroom: Part of a balanced approach to writing instruction for students with disabilities. Focus on Exceptional Children, v. 35, n. 7, p. 1, 2003.

JOHNSTON, M. V. Plasticity in the developing brain: implications for rehabilitation. Developmental disabilities research reviews, v. 15, n. 2, p. 94-101, 2009.

KNIGHT, V. F. et al. Using systematic instruction and graphic organizers to teach science concepts to students with autism spectrum disorders and intellectual disability. Focus on autism and other developmental disabilities, v. 28, n. 2, p. 115-126, 2013.

LLAUSAS, R. V. Avaliação de uma proposta de ensino de leitura e escrita para jovens e adultos utilizando software educativo. 2008. 133 f. Dissertação (Mestrado em Educação), Pontifícia Universidade Católica de São Paulo, São Paulo, 2008. 
LLOYD, B. P.; KENNEDY, C. H. Assessment and treatment of challenging behaviour for individuals with intellectual disability: a research review. Journal of Applied Research in Intellectual Disabilities, v. 27, n. 3, p. 187-199, 2014.

MANNERKOSKI, M. et al. Subjects with intellectual disability and familial need for full-time special education show regional brain alterations: a voxel-based morphometry study. Pediatric Research, v. 66, n. 3, p. 306-311, 2009.

MARTINEZ-MORGA, M.; MARTINEZ, S. Brain Development and Plasticity. Revista de Neurología, v. 62, n. 1, p. 3-8, 2016.

MARTINEZ-MORGA, M.; MARTINEZ, S. Neuroplasticity: Synaptogenesis During Normal Development and Its Implication in Intellectual Disability. Revista de Neurología, v. 64, n. 1, p. 45-50, 2017.

MAULIK, P. et al. Prevalence of intellectual disability: a meta-analysis of population-based studies. Research in Developmental Disabilities, v. 32, n. 2, p. 419-436, 2011.

MENDONÇA, M. M. Retardo Mental. In FONSECA, L. F.; PIANETTI, G.; XAVIER, C. C. Compêndio de Neurologia Infantil. Belo Horizonte: Editora Médica e Científica Ltda. (MEDSI), 2002.

MILIAN, Q. G. et al. Deficiência intelectual: doze anos de publicações na base SciELO. Revista de Psicopedagogia, São Paulo, v. 30, n. 90, p. 64-73, 2013.

OBRZUT, J. E.; HYND, G. W. Child Neuropsychology: clinical practice. Orlando: Academic Press, 2013.

OLIVEIRA, M. A.; PENARIOL, C. P.; GOYOS, C. Ensino da aplicação de tarefas de matching-to-sample computadorizadas para ensino de leitura. Acta Comportamentalia, Guadalajara, v. 21, n. 1, p. 53-67, 2013.

PICKER, J. D.; WALSH, C. A. New innovations: therapeutic opportunities for intellectual disabilities. Annals of Neurology, v. 74, n. 2, p. 382-390, 2013.

PIMENTEL, S. C. Adaptações curriculares para estudantes com deficiência intelectual na escola regular: proposta para inclusão ou para segregação? Cadernos de Educação, Pelotas, v. 45, p. 45-50, 2013.

PHILLIPS, C. Lifestyle modulators of neuroplasticity: how physical activity, mental engagement, and diet promote cognitive health during aging. Neural Plasticity, v. 2017, 2017.

PLETSCH, M. D. Repensando a inclusão escolar de pessoas com deficiência mental: diretrizes políticas, currículo e práticas pedagógicas. 2009. 254 f. Tese (Doutorado em Educação) - Centro de Educação e Humanidades, Universidade do Estado do Rio de Janeiro, Rio de Janeiro, 2009.

PLETSCH, M. D.; GLAT, R. A escolarização de alunos com deficiência intelectual: uma análise da aplicação do Plano de Desenvolvimento Educacional Individualizado. Linhas Críticas, v. 18, n. 35, p. 193-208, 2012.

POWER, J. D.; SCHLAGGAR, B. L. Neural plasticity across the lifespan. Wiley Interdisciplinary Reviews: Developmental Biology, v. 6, n. 1, 2017. 
http://dx.doi.org/10.5902/1984686X31119

REFUNDINI, D. C MARTINS, M. A.; CAPELLINI, S. A. Treinamento da correspondência grafema-fonema em escolares de risco para a dislexia. Revista Psicopedagogia, v. 27, n. 83, p. 191-201, 2010.

ROSSIT, R. A. S.; GOYOS, C. Deficiência intelectual e aquisição matemática: currículo como rede de relações condicionais. Psicologia Escolar e Educacional, v.13, n. 2, p. 213225, 2009.

ROSSIT, R. A. S.; ZULIANI, G. Repertórios acadêmicos básicos para pessoas com necessidades especiais. Temas em Psicologia, Ribeirão Preto, v. 11, n. 2, p. 114-121, 2003.

SADATO, N. et al. Tactile discrimination activates the visual cortex of the recently blind naive to Braille: a functional magnetic resonance imaging study in humans. Neurosci Lett, v. 359, p. 49-52, 2004.

SANTOS, S. C. E. S. Ensino de leitura e escrita para aprendizes com deficiência intelectual. 2012. 105 f. Dissertação (Mestrado em Educação) - Pontifícia Universidade Católica de São Paulo, São Paulo, 2012.

SANTOS, T. C. C; MARTINS, L. A. R. Práticas de professores frente ao aluno com deficiência intelectual em classe regular. Rev. bras. educ. espec, v. 21, n. 3, p. 395-408, 2015.

SIDMAN, M. Reading and auditory-visual equivalences. Journal of Speech, Language, and Hearing Research, v. 14, n. 1, p. 5-13, 1971.

SILVA, A. M. Psicologia e inclusão escolar: novas possibilidades de intervir preventivamente sobre problemas comportamentais. 2010. 151 f. Tese (Doutorado em Educação Especial). Universidade Federal de São Carlos, São Carlos, 2010.

SILVA, N. C.; CARVALHO, B. G. E. Understanding the process of school inclusion in brazil from the perspective of teachers: an integrative review. Revista Brasileira de Educação Especial, v. 23, n. 2, p. 293-308, 2017.

SNELL, M. et al. Twenty years of communication intervention research with individuals who have severe intellectual and developmental disabilities. American Journal on Intellectual and Developmental Disabilities, v. 115, n. 5, p. 364-380, set. 2010.

SOUZA, F. S.; BATISTA, C. G. Indicadores de Desenvolvimento em Crianças e Adolescentes com QI Igual ou Inferior a 70. Rev. bras. educ. espec, v. 22, n.4, p. 493-510, 2016.

SOUZA, M. C.; GOMES, C. Neurociência e o déficit intelectual: aportes para a ação pedagógica. Revista Psicopedagogia, São Paulo, v. 32, n. 91, p. 104-114, 2015.

STROMER, R.; MACKAY, H.; STODDARD, L. Classroom applications of stimulus equivalence technology. Journal of Behavioral Education, v. 2, n. 3, p. 225-256, dec. 1992.

TANNOUS, G. S. Inclusão do aluno com deficiência mental : experiências psicossociais dos professores da escola pública. 2005. 174f. Dissertação (Mestrado em Psicologia). Universidade Católica Dom Bosco, São Paulo, 2005.TEBEROSKY, A. e PALÁCIO, M. G. Os processos de leitura e escrita: novas perspectivas. Porto Alegre: ARTMED, 1987. 
THAMBIRAJAH, M. S. Developmental assessment of the school-aged child with developmental disabilities: A clinician's guide. London: Jessica Kingsley Publishers, 2011.

VASCONCELOS, M. M. A neurobiologia do retardo mental. Jornal de Pediatria, Rio de Janeiro, v. 80, n. 2, p. 71-82, 2004.

VELTRONE, A. A. Inclusão escolar do aluno com deficiência intelectal no estado de São Paulo: indentificação e caracterização. 2008. 193f. Tese (Doutorado em Educação Especial) - Centro de Educação e Ciências Humanas, Universidade Federla de São Carlos, São Carlos, 2008.

VELTRONE, A. A.; MENDES, E. G. Caracterização dos profissionais responsáveis pela identificação da deficiência intelectual em escolares. Revista de Educação Especial, Santa Maria, v. 24, n. 39, p. 61-76, 2011.

VOSS, M. W. et al. Bridging animal and human models of exercise-induced brain plasticity. Trends Cognitive Science., v. 17, n. 10, p. 525-544, 2013.

\section{Correspondência}

Patricia Martins de Freitas - Universidade Federal de Minas Gerais. Rua: Rodovia Av. Pres. Antônio Carlos, 6627 - Pampulha, Belo Horizonte. CEP: 31270-901. Belo Horizonte, Minas Gerais, Brasil.

This work is licensed under a Creative Commons Attribution-NonCommercial 4.0 International (CC BY-NC 4.0) 